\title{
Partial reduction of microglia does not affect tau pathology in aged mice
}

\author{
Rachel E. Bennett ${ }^{1}$, Annie Bryant ${ }^{1}$, Miwei Hu', Ashley B. Robbins ${ }^{1}$, Sarah C. Hopp ${ }^{2,3^{*}+}$ (D) and Bradley T. Hyman ${ }^{1+}$
}

\begin{abstract}
Background: Activation of inflammation pathways in the brain occurs in Alzheimer's disease and may contribute to the accumulation and spread of pathological proteins including tau. The goal of this study was to identify how changes in microglia, a key inflammatory cell type, may contribute to tau protein accumulation and pathologyassociated changes in immune and non-immune cell processes such as neuronal degeneration, astrocyte physiology, cytokine expression, and blood vessel morphology.

Methods: We used PLX3397 (290 mg/kg), a colony-stimulating factor receptor 1 (CSF1R) inhibitor, to reduce the number of microglia in the brains of a tau-overexpressing mouse model. Mice were fed PLX3397 in chow or a control diet for 3 months beginning at 12 months of age and then were subsequently analyzed for changes in blood vessel morphology by in vivo two-photon microscopy and tissues were collected for biochemistry and histology.

Results: PLX3397 reduced microglial numbers by 30\% regardless of genotype compared to control diet-treated mice. No change in tau burden, cortical atrophy, blood vessels, or astrocyte activation was detected. All Tg4510 mice were observed to have an increased in "disease-associated" microglial gene expression, but PLX3397 treatment did not reduce expression of these genes. Surprisingly, PLX3397 treatment resulted in upregulation of CD68 and Tgfi $\beta$.

Conclusions: Manipulating microglial activity may not be an effective strategy to combat tau pathological lesions. Higher doses of PLX3397 may be required or earlier intervention in the disease course. Overall, this indicates a need for a better understanding of specific microglial changes and their relation to the disease process.
\end{abstract}

Keywords: Microglia, Alzheimer's disease, Tau

\section{Introduction}

An increase in the number of reactive microglia is a key feature of Alzheimer's disease (AD, [35]), with data from multiple genome-wide association studies confirming these cells are important to disease pathogenesis $[9,40]$. In particular, increased numbers of microglia are frequently found both surrounding amyloid $\beta(A \beta)$ plaques and in brain regions where neurofibrillary tangles containing tau are present $[3,34,36]$. The role of microglia near these pathological lesions is believed to aid clearance of

\footnotetext{
*Correspondence: hopps1@uthscsa.edu

${ }^{\dagger}$ Sarah C. Hopp and Bradley T. Hyman contributed equally to this work.

${ }^{2}$ Biggs Institute for Alzheimer's and Neurodegenerative Disease, University of Texas Health Science Center San Antonio, 7703 Floyd Curl Drive, San Antonio, TX 78229, USA

${ }^{3}$ Department of Pharmacology, University of Texas Health Science Center San Antonio, San Antonio, TX 78229, USA

Full list of author information is available at the end of the article
}

toxic protein aggregates [27, 28]. On the other hand, microglia may influence neurodegenerative phenotypes including tau spreading and synaptic loss, and microglial secretion of inflammatory factors may exacerbate pathology $[5,11,19,22,24,32]$. Microglia activation induces neuronal tau phosphorylation via p38 MAPK [30], and reduction of microglia activation is capable of reducing neuronal tau phosphorylation in vivo [16]. Taken together, it is unclear how microglia influence AD. Recent studies have identified gene expression changes in microglia from Alzheimer's disease brain and mouse models indicating that the subset of microglia near plaques are phenotypically distinct from non-plaque-associated microgliahighlighting the importance of understanding the diversity of responses to disease pathology $[18,21]$.

To better understand if microglia change neurofibrillary tangle pathology, we investigated the relationship

(C) The Author(s). 2018 Open Access This article is distributed under the terms of the Creative Commons Attribution 4.0 International License (http://creativecommons.org/licenses/by/4.0/), which permits unrestricted use, distribution, and 
between the presence of microglia, tau protein accumulation, and other tau-related pathological events. We have previously observed changes in blood vessel morphology that take place between 12 to 15 months of age and hypothesized that microglia-mediated inflammatory pathways may play a role at this time point [4]. Microglia are capable of phagocytosing tau aggregates and have been hypothesized to contribute to the pathological spread of tau in the brain by inefficient degradation of tau seeds [14]. Bhaskar et al. found that mice lacking a microglial CX3CR1 fractalkine receptor had enhanced tau phosphorylation via increased activity of p38 MAPK [5, 30]. Separately, others have used clodronate liposomes or the colony-stimulating factor 1 receptor (CSF1R) inhibitors PLX3397 or PLX5622 to deplete microglia from the brains of mouse models of AD. In one study of early tau accumulation, depletion of microglia in mice expressing tau in entorhinal cortex reduced pathological tau spread along axons to the hippocampal dentate gyrus as well as AT8 tau phosphorylation in young PS19 mice [1]. In a separate model, in aged 3xTg mice which develop both $A \beta$ plaques and tangles, microglial depletion appeared to have a minimal effect on measures of $A \beta$ plaque pathology and no effect on tau pathology assessed by either total tau or AT8 phosphorylation [8]. In another AD mouse model, such as the 5XFAD line which only develops plaque pathology, early microglia depletion reduces pathological accumulation of $\mathrm{A} \beta$ [37] but later depletion does not, and only has a modest protective effect on neurons [38].

In light of these observations of the complex role of microglia in $\mathrm{AD}$ mouse models, we sought to address two questions. First, we asked if tau pathology induces "disease-associated" microglia gene expression changes similar to those reported for microglia in $\mathrm{A} \beta$ plaque depositing mice $[18,21]$. This was of particular interest due to the apparent resistance of plaque-associated microglia to elimination by CSF1R inhibitors: in one study, PLX3397 depleted 80-90\% of total microglia throughout the brain, but only $50 \%$ of plaque-associated microglia [38]. Second, we hypothesized that depletion of microglia with PLX3397 in aged mice with tau pathology may reduce pathological features of tau accumulation, particularly in light of the role of microglia in tau phosphorylation, tau accumulation, astrocyte activation, and blood vessel pathology [4], which are all features of the $\operatorname{Tg} 4510$ mouse model.

\section{Methods}

\section{Animals}

All mice used in these studies were housed and maintained under the McLaughlin Research Institute or Massachusetts General Hospital Animal Use \& Care Committee. In total, $33 \mathrm{Tg} 4510$ (129S6.Cg-Tg(Camk2a-tTA)1Mmay/Jlws); FVB$\mathrm{Tg}($ tetO-MAPT*P301L)Kha/JlwsJ) and litter-mate controls
(129S6-Tg(Camk2a-tTA)1Mmay/JlwsJ) were used in these studies and were originally obtained from Jackson Laboratory (Bar Harbor, ME) [33]. PLX3397 was synthesized by Plexxikon and custom blended with a commercially available formulation enriched for vitamins (TK-97184, Teklad). Mice were randomly assigned PLX3397 (PLX; 290 mg/kg) diet or control diet at 12 months of age and each group was balanced for gender. Mice were maintained on each diet for 3 months.

\section{Histology}

Prior to slicing, fixed hemispheres were equilibrated in $30 \%$ sucrose in PBS for $24 \mathrm{~h}$. Then, hemispheres were frozen and coronally sliced on a freezing microtome. Ten sets of $40-\mu \mathrm{m}$-thick sections were collected such that each set contained sections at $400 \mu \mathrm{m}$ intervals. Sections were stored in $30 \%$ glycerol in PBS at $-20{ }^{\circ} \mathrm{C}$ until use. For Iba-1 immunohistochemistry, sections were rinsed in Tris-buffered saline (TBS), incubated in $0.3 \%$ hydrogen peroxide for $10 \mathrm{~min}$, then blocked in 3\% normal goat serum (NGS) in $0.25 \%$ Triton-x TBS (TBS-X) for $30 \mathrm{~min}$ prior to incubation in goat anti-Iba-1 (1:1000, WAKO) overnight at $4{ }^{\circ} \mathrm{C}$ in blocking solution. The following day, sections were washed in TBS, incubated in donkey anti-goat IgG in $0.25 \%$ TBS-X for $1 \mathrm{~h}$, then washed and incubated with streptavidin-HRP (Vector Labs ABC) for $30 \mathrm{~min}$, and subsequently washed and developed with diaminobenzidine containing $\mathrm{NiCl}_{2}$ for 4-5 min. Following staining, sections were washed, mounted on glass slides, and then dehydrated in ascending ethanol/xylene and coverslipped with Cytoseal XYL (Thermo Fisher Scientific). For triple labeling, sections were treated as before then incubated in rabbit anti-NeuN (1:1000, Millipore), mouse anti-tau (AT8, 1:1000, Thermo Fisher Scientific), and chicken anti-GFAP (1:1000, Millipore) in blocking solution overnight at $4{ }^{\circ} \mathrm{C}$. The following day, sections were incubated for $1 \mathrm{~h}$ with goat anti-mouse IgG Alexa488, goat anti-rabbit IgG Alexa 555, and goat anti-chicken IgG Alexa 647 (1:2000, Thermo Fisher Scientific) in TBS-X. Sections were then washed and mounted on glass slides and allowed to dry prior to coverslipping with Fluoromount G (Southern Biotech).

\section{Stereology and image analysis}

For stereological quantification of Iba-1 positive microglia, a brightfield microscope fitted with a motorized stage was used along with computer-assisted stereological toolbox version 2.3.1.5 (Olympus America). Slides were first viewed with $\mathrm{a} \times 1.25$ objective to identify the anterior-most section in each series containing hippocampus and dentate gyrus was chosen as the first section for analysis and following two posterior sections were also included for a total of three slices. Dorsal cortex was then outlined beginning at midline and extending 
laterally to the level of the dorsal thalamus. This ROI was chosen as it overlapped best with two-photon measures. In total, $8 \%$ of the total area of these regions was counted via stereological random sampling. In a separate set of sections, for stereological analysis of NeuN, $1 \%$ of the area was sampled and for AT8, 8\% of the area was sampled while the images were viewed by epifluorescence. These sections were also imaged using a VS120 Olympus slide scanner to quantify GFAP (Alexa 647). A threshold-based ImageJ approach was used to quantify the total GFAP + as a percent of the total ROI. Briefly, a DAPI image was used to outline cortex as described above and then the "moments" threshold was applied to all GFAP images. The thresholded GFAP area and total outlined area was then measured per image.

For analysis of blood vessel density and length from two-photon z-stack images, all processing was done in Image as described previously. In short, the 3D Objects Counter was used to measure blood vessel volume and the Skeletonize (2D/3D) plugin was used to measure blood vessel length. All measures were normalized to average cortical thickness (measured from Iba-1 stained sections) to account for atrophy.

\section{Western blots}

To prepare brain protein homogenates, the cerebellum and brain stem was removed and the remaining hemisphere was placed in a $2 \mathrm{ml}$ glass dounce homogenizer with $200 \mu \mathrm{l}$ PBS containing protease inhibitors (Complete Mini, Roche). Tissue was homogenized with 30 up/down strokes. The homogenate was then centrifuged at $3000 \times g$ and the supernatant was reserved for analysis. For Western blotting, $10 \mu \mathrm{g}$ of protein was boiled for $5 \mathrm{~min}$ in SDS reducing agent and loading dye (Invitrogen) and then was loaded on a $4 \%$ to $12 \%$ bis-tris SDS-PAGE in MES buffer (Invitrogen). Samples were run at $120 \mathrm{~V}$ for $90 \mathrm{~min}$ then transferred to nitrocellulose. Membranes were blocked using Odyssey blocking reagent (Li-Cor) for $30 \mathrm{~min}$ then incubated overnight in primary antibodies. Antibodies used in these experiments included mouse anti-tau (AT8, 1:1000, Thermo Fisher Scientific), rabbit anti-phospho tau T231 (1:1000, Invitrogen), chicken anti-Gapdh (1:5000, Millipore), mouse anti-p38 MAPK (1:1000, Cell Signaling Technology), and rabbit anti-phospho-p38 MAPK (1:1000, Cell Signaling Technology). Infrared-labeled secondary antibodies (1:5000, Li-Cor) were then incubated with membranes and detected with a Li-Cor imaging system.

\section{Cell-based tau seeding activity assay}

To assess the presence of bioactive tau "seeds," we used a fluorescence resonance energy transfer (FRET) cell-based assay [13]. In this assay, HEK293 cells stably express the tau repeat domain (TauRD) conjugated to a yellow fluorescent protein (YFP) or cerulean fluorescent protein
(CFP). CFP to YFP FRET at $535 \mathrm{~nm}$ can be measured within these cells using flow cytometry after application of tau aggregates or "seeds." For the experiments herein, cells were plated at 20,000 cells per well in a 96-well plate coated with poly-D-lysine (PDL; $50 \mu \mathrm{g} / \mathrm{ml}$, Sigma) for at least $3 \mathrm{~h}$. The day after plating, brain lysate from $\mathrm{rTg} 4510$ or WT mice was added to the wells at $0.5 \mu \mathrm{g} /$ well in Opti-MEM medium (\#11058-021, Life Technologies) with 1\% Lipofectamine 2000 (\#11668019, Life Technologies) to promote internalization of tau seeds. Twenty-four hours later, cells were detached with trypsin, transferred to round-bottom plates, centrifuged at $300 \mathrm{~g}$, fixed with $2 \%$ paraformaldehyde (PFA) in suspension for $10 \mathrm{~min}$, centrifuged at $300 \mathrm{~g}$, and then finally resuspended in phosphate-buffered saline (PBS). Fixed cells were then analyzed on a MACSQuant VYB (Miltenyi Biotec) flow cytometer with excitation with a $405 \mathrm{~nm}$ laser and emission captured by a $525 / 20 \mathrm{~nm}$ band pass filter. Gates were drawn to select live cells by forward and side scatter channels and subsequently single cells (singlets) by forward scatter area and height. Then, 20,000 singlets were collected for analysis. Integrated FRET density (IFD) was calculated by multiplying the median fluorescence intensity of FRET-positive singlets by the percentage of FRET-positive events within the singlets gate. Each flow condition was performed in at least triplicate.

\section{Quantitative PCR}

RNA was obtained using the Qiagen RNeasy Mini Kit (Qiagen Cat. No. 74104). All contact surfaces were cleaned with 70\% Ethanol and RNaseZAP (Sigma-Aldrich, Cat. No. R2020). Tissue was homogenized with a mortar and pestle over dry ice and $30 \mathrm{mg}$ was weighed and separated into a $1.5 \mathrm{ml}$ RNase, DNase free Eppendorf tube on ice. Further, $600 \mu \mathrm{l}$ of lysis buffer (RLT buffer, Qiagen) was added to each tube and the samples was sonicated at $10 \%$ amplitude for $30 \mathrm{~s}$. Samples were then spun at 13,000 rpm for 3 min and the supernatant was transferred to a new tube for RNA isolation. On-column RNA isolation was performed according to the RNeasy Mini Kit manufacturer's instructions and the final RNA sample was eluted in $30 \mu \mathrm{l}$ of DNase, RNase free water. Final yield was quantified using a Nanodrop spectrophotometer.

All cDNA synthesis reactions were performed with $100 \mathrm{ng}$ of total RNA per sample using the QuantiTect Reverse Transcription Kit (Qiagen Cat. No. 205311). The cDNA synthesis reactions were then added to a 96-well plate with QuantiTect SYBR Green Mastermix (Qiagen Cat. No. 204143), sealed with optically clear flat seal caps and spun at $300 \times$ g briefly. All qPCR reactions were performed in a Bio-Rad CFX96 real-time cycler [15 min at $95{ }^{\circ} \mathrm{C}, 40$ cycles $\left(15 \mathrm{~s}\right.$ at $94{ }^{\circ} \mathrm{C} ; 30 \mathrm{~s}$ at $55^{\circ} \mathrm{C}$; $30 \mathrm{~s}$ at $\left.72{ }^{\circ} \mathrm{C}\right)$ ]. QuantiTect primer assays were used for 
the following gene targets: Actb, Gapdh, Hprt, Icam1, Icam2, Tjp1, Cldn5, Ocln1, Vcam1, Il-beta, Serpine1, and Plau (Qiagen, Product No. 249900).

A custom TaqMan Fast 96-well qPCR array plate (Applied Biosystems) was designed to examine six microglia genes based on current literature highlighting a specific neurodegenerative phenotype found in microglia in $\mathrm{AD}$ and other neurodegenerative mouse models: Cx3cr1, Apoe, Trem2, Il1b, CD68, and Tgfb1. The arrays used Gapdh as a reference gene and 18s rRNA as an internal control. Each plate contained a TaqMan probe and PCR primer set dried down in each well. cDNA was added to each well diluted in 2x TaqMan Fast Universal PCR master mix (Applied Biosystems, 4352042) and ultrapure water. Plates were analyzed on a Bio-Rad CFX96 qPCR machine; Enxymes were activated for $20 \mathrm{~s}$ at $95{ }^{\circ} \mathrm{C}$, then 40 cycles of denaturing $\left(95{ }^{\circ} \mathrm{C}, 3 \mathrm{~s}\right)$ and annealing/extending $\left(30 \mathrm{~s}, 60^{\circ} \mathrm{C}\right)$ followed by data collection were performed.

\section{In vivo imaging of blood vessels}

At 15 months, acute cranial windows were performed as described previously [4]. Mice were injected with intravenous $70 \mathrm{kDa}$ dextran conjugated to Texas Red to image blood vessels. All two-photon images were acquired over the somatosensory and motor cortex using an Olympus two-photon imaging system equipped with a water immersion lens (25x, N.A. $=1.05)$ and a Ti:Sapphire (MaiTai, Spectra Physics) tunable laser at $900 \mathrm{~nm}$. For each mouse, six randomly sampled regions of interest (ROIs) were acquired (three per hemisphere). Each ROI was acquired at $3 \times$ digital zoom, beginning $50-100 \mu \mathrm{m}$ below the surface of the brain to avoid surgery artifacts. A total of 50 slice images were captured at $2 \mu \mathrm{m}$ steps per ROI. Immediately following imaging, mice were euthanized and perfused with $10 \mathrm{ml}$ cold PBS. One hemisphere was drop-fixed in $4 \%$ paraformaldehyde and the other hemisphere was snap frozen and stored at $-80{ }^{\circ} \mathrm{C}$ for biochemistry/RNA analysis.

\section{Statistics}

All data were first confirmed to be normally distributed using Shapiro-Wilkes normality test. For ELISA data analyses, a two-tailed student's $t$ test was used. In all other cases, a two-way ANOVA was used to determine overall significance of genotype and treatment followed by post-hoc Sidak's multiple comparisons. A threshold of $p<0.05$ was used to determine significance.

\section{Results}

\section{Reduction of microglia in Tg4510 mice after CSF-1r} blockade

Beginning at 12 months of age, $\operatorname{Tg} 4510$ mice and littermate wild-type controls were fed chow containing PLX3397 (290 mg/kg, PLX3397) or control diet (Fig. 1a-c) for 3 months. By 15 months of age, Tg4510 mice on a control diet had an approximate $45 \%$ increase in the total number of Iba-1 positive microglia per cubic millimeter of cortical tissue compared to wild-type mice (wild-type $5975 \pm 1060$ microglia per $\mathrm{mm}^{3}$, Tg4510 8465 \pm 1167 microglia per $\mathrm{mm}^{3}$; overall two-way ANOVA effect of genotype $p<0.001$, treatment $p<0.001$, interaction $p=7324$; PLX-WT vs PLX-TG $p=0.012$ ). Treatment of mice with PLX3397 resulted in a 30\% reduction in the number of microglia in Tg4510 $(5956 \pm 1490$ microglia per $\left.\mathrm{mm}^{3} ; p=0.007\right)$ and wild-type mice (3624 \pm 1885 microglia per $\mathrm{mm}^{3} ; p=0.02$ ) compared to mice fed control diet. Notably, Tg4510 mice treated with PLX3397 had a density of microglia comparable to untreated wild-type mice $(p>0.999)$ though surprisingly, treating with this dose did not result in the $>96 \%$ elimination of microglia previously reported [10]. In total, we treated 16 mice with PLX3397 in this study and only observed 1 individual to have a $>96 \%$ depletion of microglia (Fig. 1b). Notably, this wild-type mouse was observed to have a greater body weight than cage mates and we postulate that it consumed a higher dose over the duration of the experiment. In general, our results are similar to those achieved by Dagher et al. in aged 3xTg mice treated with a related compound PLX5622 who also reported a $30 \%$ reduction in microglia between treated and untreated mice [8].

We hypothesized that one explanation for this discrepancy in effective dose could be related to changes in CSF1R expression, the target of PLX3397, with age and pathological tau accumulation. RNA was extracted and probed for CSF1R expression by quantitative PCR. We did not detect a change in relative expression levels of CSF1R between treated and untreated mice (Fig. 1d, two-way ANOVA $p=0.400)$, between genotypes $(p=$ 0.408 ), or between 6 - and 15-month-old mice (Fig. 1e, two-way ANOVA effect of age $p=0.273$ ).

\section{Disease-associated microglial profiles in Tg4510 mice}

Next, we examined gene expression from the brains of mice treated with PLX3397 to determine how the setting of inflammation is altered by treatment. Recently, others have identified "disease-associated" transcription profiles in mice that develop amyloid $\beta$ plaques and we sought to include key markers of this microglial sub-type including Apoe and Trem2, in our analysis. Gene expression from total brain extracts indicate that expression levels of Apoe, Cd68, Cx3cr1, Ill $\beta, T g f \beta 1$, and Trem2 were all significantly upregulated in Tg4510 mice versus wild-type (Fig. 1f). Cd68 (macrosialin) is a cell surface receptor that is upregulated in phagocytic microglia and in Alzheimer's disease. In Tg4510 mice treated with PLX3397, Cd68 was significantly upregulated compared to control diet. Tgf $\beta$ is a protein involved in maintaining microglial homeostasis and was also upregulated in 


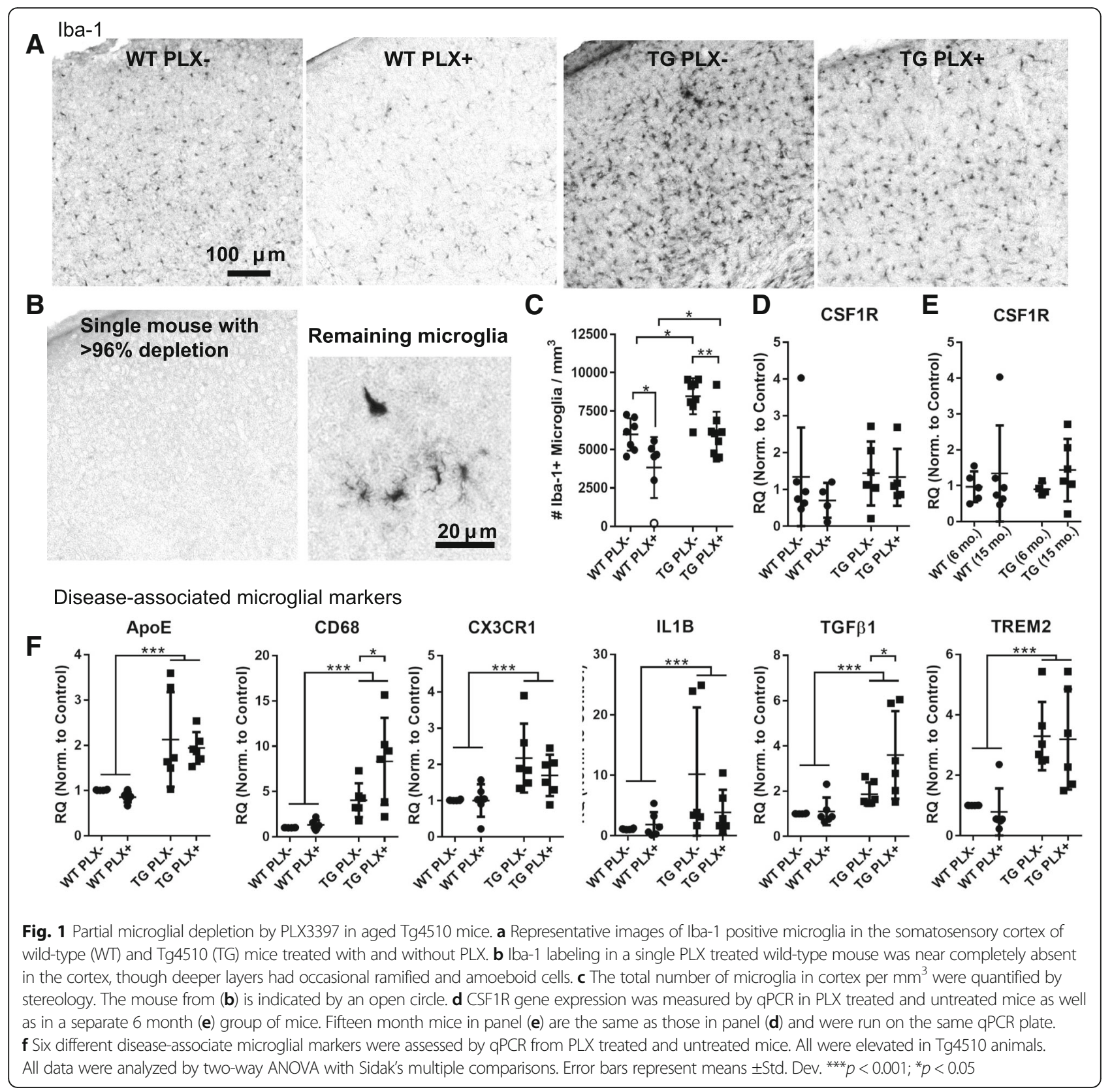

Tg4510 mice fed PLX3397 versus control diet. Altogether, these data indicate that tau expression also upregulates a phenotype similar to that of amyloid induced pattern of "disease-associated" microglial gene expression and that reducing microglial numbers does not eliminate these gene changes.

Tau pathology and phosphorylation is unaltered

Having confirmed that we altered microglial density and gene expression via PLX3397 treatment, we next determined the effect of this manipulation on tau. Notably, microglia may play a role in spread of pathological tau from one brain region to another [1] as well as contribute to the post-translational modification of tau through kinase activity and phosphorylation [24, 29]. To explore this, we used a FRET-based HEK cell tau seeding assay to determine if reducing microglia also reduced the potential of tau to seed new aggregates through templated misfolding (Fig. 2a, b). Protein lysates from Tg4510 brain were capable of inducing FRET aggregates but not protein from wild-type brain as shown previously (two-way ANOVA, effect of genotype $p<0.0001)$. No difference was detected in the seeding activity of tau from PLX + and PLX - mice ( $p=$ 0.6122). We confirmed that PLX treatment did not alter tau protein levels by randomly sampling Tg4510 mice 


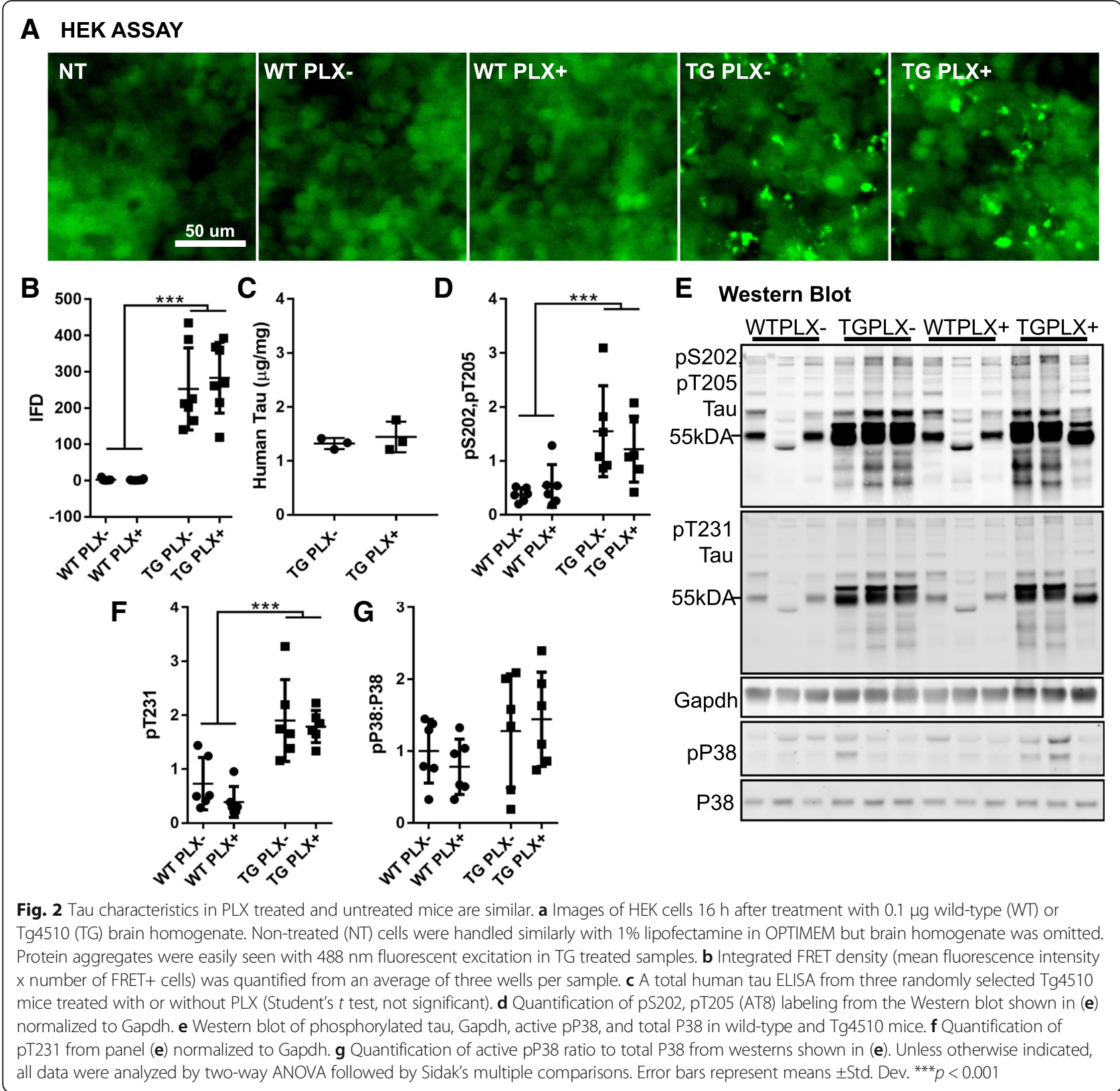

from each group and measuring total tau by ELISA (Fig. 2c, Student's $t$ test $p=0.525$ ).

We then looked at the phosphorylation state of tau in these mice, focusing on phosphorylation sites pS202/T205 and pT231 as these may be enhanced by active p38. While phosphorylation was increased in $\operatorname{Tg} 4510$ mice compared to wild-type, no difference was detected between PLX+ and PLX- mice (Fig. 2d-f). Similarly, when we assess active phospho-P38 by Western blot, no increase in the total ratio of pP38:total P38 was observed indicating that this does not play a significant role in this model at this age (Fig. 2e, g).
We further confirmed these biochemical observations of tau pathology by performing tau immunofluorescence in tissue sections using the antibody AT8 (pS202, T205; Fig. 3a, c). The total number of tau+ somas in the cortex was counted by stereology which confirmed no change in the number of AT8+ neurons in cortex (two-way ANOVA effect of genotype $p<$ 0.001, TG PLX+ vs. PLX $-p=0.899$ ).

\section{No change in neuron loss or count}

Cortical atrophy is ongoing in $\operatorname{Tg} 4510$ mice at this age [4]. In accord with these observations, stereological counts of NeuN positive neurons in the cortex revealed 


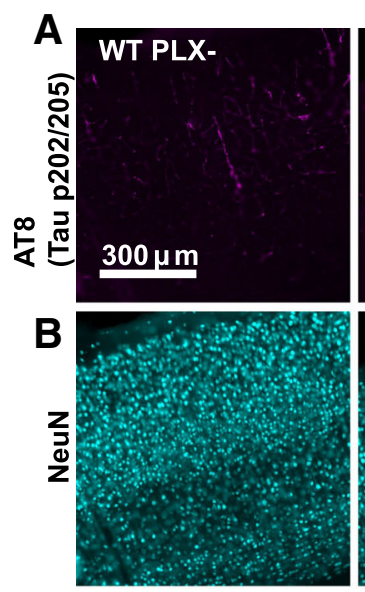

C

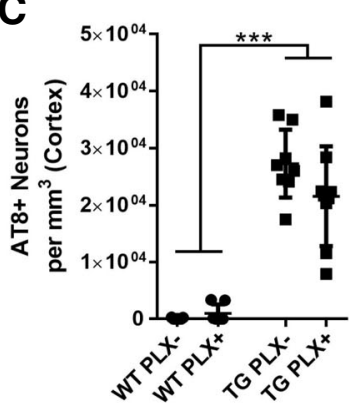

WT PLX+
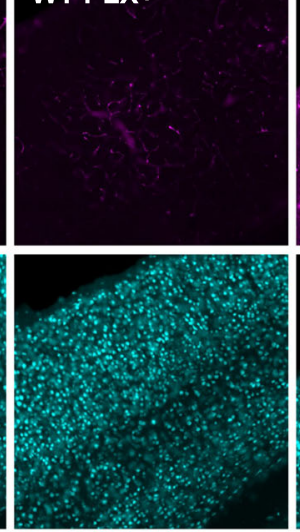

D
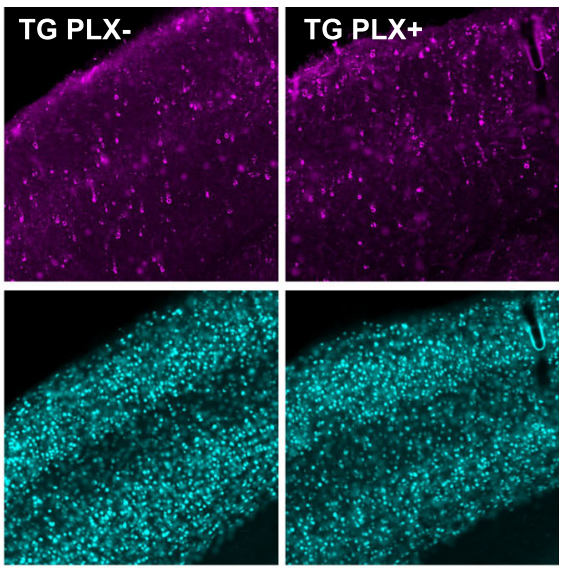

E
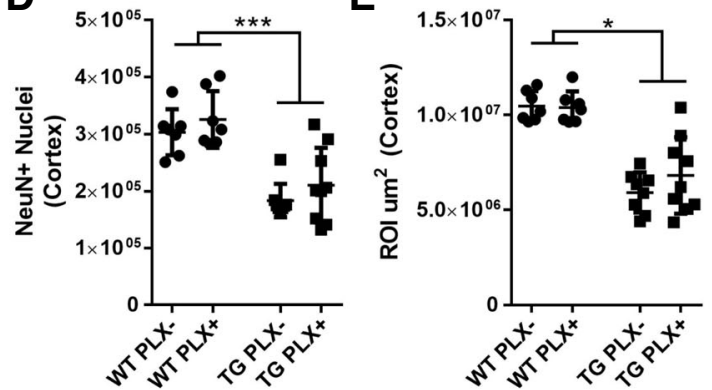

Fig. 3 No change in location of tau deposition or neuronal loss. a Tau (pS202, pT205; AT8) labeling in somatosensory cortex of wild-type (WT) and Tg4510 (TG) mice. b NeuN labeling of neuronal nuclei in the same area of cortex. c AT8 quantification of the number of neuronal somas per cubic $\mathrm{mm}$ by stereology. $\mathbf{d}$ The total number of NeuN nuclei in cortex estimated by stereology. e The total region of interest (ROI) area per cubic micron measured for these analyses. All data were analyzed by two-way ANOVA. Error bars represent \pm Std. Dev. ${ }^{* * *} p<0.001 ;{ }^{*} p<0.05$

neuronal number in the cortex was reduced by approximately $50 \%$ compared to wild-type mice by 15 months. To determine if reducing microglia may exacerbate or slow neurodegeneration process, we counted NeuN neurons in Tg4510 mice treated with PLX; no change was detected in the number of neurons observed in cortex in PLX+ versus PLX- Tg4510 mice (two-way ANOVA, effect of genotype $p<0.001$ ).

\section{No change in blood vessel measures}

We have recently reported that blood vessel morphology is distorted in the cortex of aged Tg4510 mice with capillary density increasing between 12 and 15 months of age. To assess the possible contribution of microglia to this phenotype, we imaged blood vessels in mice via in vivo two-photon microscopy (Fig. 4a-c). Z-stack images acquired from somatosensory and motor cortex areas in PLX - and PLX+ Tg4510 mice appeared equally distorted with both groups showing similarly increased vascular density (Fig. 4b) and length (Fig. 4c) compared to wild-type mice. Further, in previous blood vessel studies, we identified gene expression changes associated with the morphological alterations. Two of the most highly upregulated genes, Plau and Serpine1, are also expressed by microglia. In these studies, we confirmed that Plau and Serpine1 are both overexpressed in Tg4510 brain compared to wild-type (Fig. 4d, e) and attempted to determine the relative contribution of microglia to this increase. No reduction in the expression of either gene was observed, and surprisingly, Plau expression was increased in PLX+ versus PLX- mice indicating that reducing microglia may either have little impact on or may exacerbate these pathological blood vessel changes in Tg4510 mice.

\section{No change in GFAP-positive astrocytes}

Finally, to determine if PLX treatment or microglial decrease to control levels had an effect on astrocyte activation, we measured GFAP in these mice (Fig. 5a, b). Tg4510 mice had considerable elevation of GFAP particularly in the cortex (two-way ANOVA effect of genotype $p<0.001)$ and underlying white matter. Quantification of GFAP in cortex revealed no change between PLX+ and PLX- Tg4510 or control mice $(p=0.3261)$.

\section{Discussion}

These studies indicate that reducing the number of microglia in the aged brain, after tangle formation and neuronal death has begun, may not prevent additional 


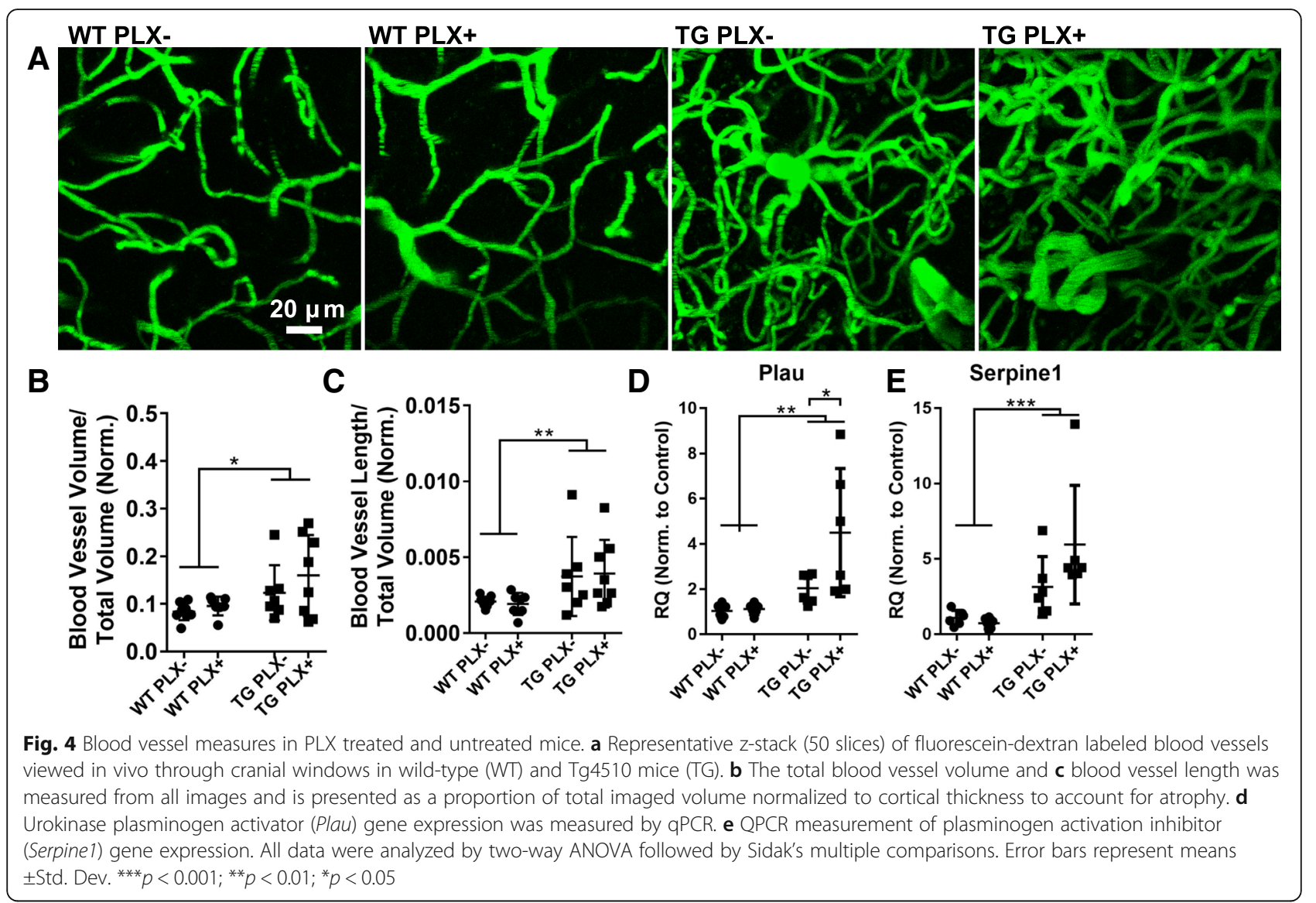

tau accumulation and related pathological changes. In these experiments, mice fed a diet containing PLX3397 were found to have a $30 \%$ reduction in the number of microglia in the cortex, so that the microglial complement of treated $\operatorname{Tg} 4510$ mice was numerically equivalent to that of controls. This intervention did not alter the extent of cortical neurodegeneration, assessed either by the number of neurons with AT8+ tau inclusions or the extent of neuronal loss, extent of astrocyte activation, or tau associated changes in vascular morphology.
Additionally, this intervention did not modify p38-MAPK activation. This was a surprising finding given the large body of literature demonstrating the role of microgliosis in activation of neuronal p38-MAPK and associated tau phosphorylation, and a growing body of literature demonstrating crosstalk between microglia and astrocytes [25] and microglia and endothelial cells [26].

Importantly, we found that genes associated with "disease-associated" microglia near plaques such as ApoE and Trem 2 are also upregulated in the brains of $\operatorname{Tg} 4510$

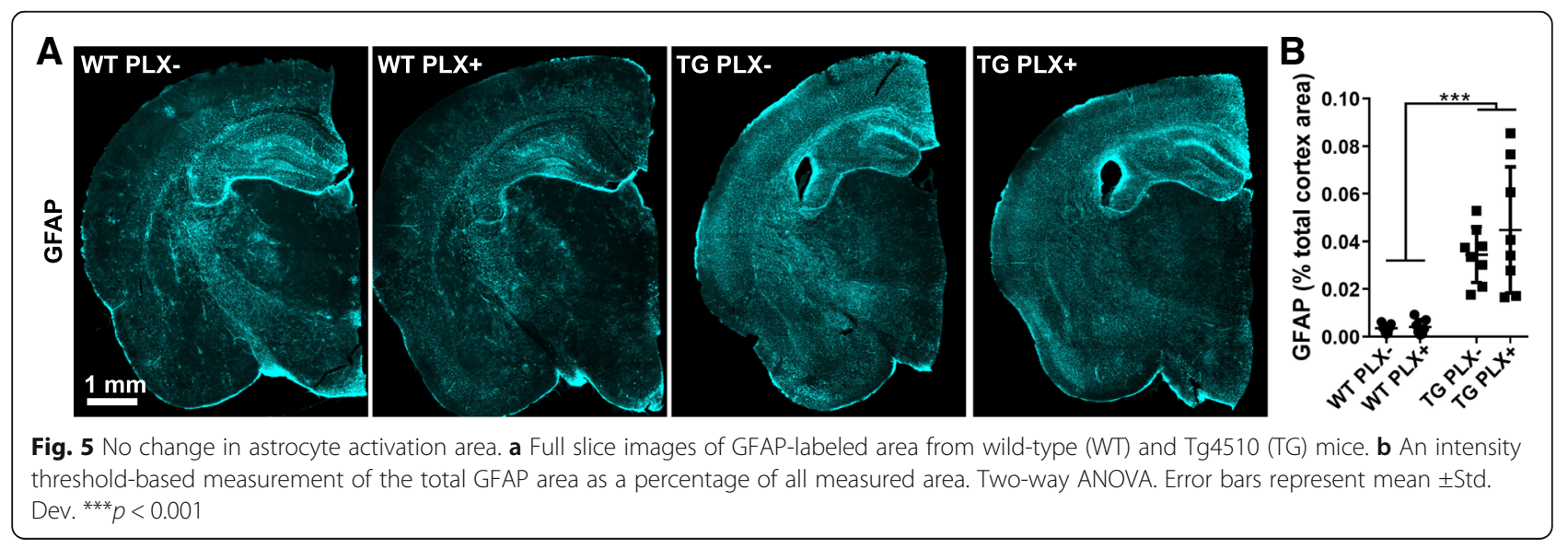


mice [18]. This subset of microglia may thus not be unique to $A \beta$ plaques. We also found that reducing the number of microglia in the brain by $30 \%$ does not result in a similar $30 \%$ reduction in the expression of these "disease-associated" microglial genes. One reason for this could include upregulation by other cell types including astrocytes in the case of Apoe, Ill $\beta$, and Tgf1 $\beta$. However, Trem2, $C x 3 \mathrm{cr} 1$, and $C d 68$ are nearly exclusively expressed by microglia in the brain. Further, despite reduction of microglia in PLX-treated mice, $C d 68$ and $\operatorname{Tg} f 1 \beta$ both were surprisingly significantly upregulated in PLX+ versus PLX- animals. This finding is not unique to the study: indeed, other studies utilizing PLX5622 to deplete microglia in the 3xTg AD mouse model have found that some inflammatory markers significantly increase despite reduced numbers of microglia, including tumor necrosis factor $\alpha$ (TNF $\alpha)$ and CXCL1 [8]. In other mouse models of neurodegeneration, depletion of microglia with PLX3397 also exacerbates inflammatory markers $[7,17,42]$, suggesting this may be a common feature of microglia depletion during neurodegeneration-possibly indicating a compensatory upregulation of inflammatory factors in response to microglial depletion in the setting of ongoing neurodegeneration. Overall, these data suggest either that the microglia that remain after depletion may change as a consequence of anti-CSF1R treatment or that some of the tau-responsive, activated microglia become less sensitive to anti-CSF1R treatment. Furthermore, if the microglia remaining after depletion are indeed more reflective of a "disease-associated" phenotype, as our data and others' suggest, this also may indicate that repopulation from these leftover microglia may not be beneficial for "resetting" since microglia are repopulated from the remaining microglia [15]. Further investigation into whether these repopulated microglia carry over similar inflammatory phenotypes, epigenetic signatures, or other cellular memory would be vital prior to therapeutic application of microglia repopulation.

Despite using a PLX3397 dose $(290 \mathrm{mg} / \mathrm{kg})$ reported to eliminate microglia from the brain, in these experiments, we did not achieve a similar level of reduction [10]. The number of microglia eliminated in aged $\operatorname{Tg} 4510$ mice was on the same order of reduction reported using a related drug PLX5622 at a similar dose $(300 \mathrm{mg} / \mathrm{kg})$ [8]. Here, it does not appear that the failure of the drug to completely eliminate microglia was due to up- or downregulation of CSF1R with aging or tau overexpression, although it is unknown if sensitivity to CSF1R blockade changes with age. Thus, considering that a single mouse in our experiment was observed to have a near complete lack of microglia, for complete elimination of microglia in future experiments a higher dose $(1200 \mathrm{mg} / \mathrm{kg})$ may be required as reported in
Dagher et al. [8]. Another limitation of this study is that peripheral monocytes were not measured. CSF1R is also expressed in macrophage, neutrophils, and osteoclasts and it is unclear if these populations were similarly altered in these mice [6, 31]. Others have reported that splenic macrophage populations remain largely intact following PLX3397 treatment, though numbers are reduced at sites of inflammation [12, 20, 23, 39]. It is unclear how these peripheral cells may interact with tau pathology and neurodegeneration in this model, though these experiments indicate that PLX treatment at this dose is unlikely to have an impact.

These results are in line with other microglial manipulation models which have similarly observed little change in pathological tau deposition. Only when microglia numbers are reduced in acute models of tau spread, or in young mice, has depletion of microglia had an effect on abrogating tau pathological spread or phosphorylation [1]. However, virally expressed tau models do not allow evaluation of other phenotypes that may be microglial associated such as neuronal death, astrogliosis, blood vessel pathology, or chronic microgliosis. Additionally, depletion of microglia in younger mouse models of neurodegeneration appears to have a larger effect on mitigating $\mathrm{AD}$ pathology overall $[1,37]$ which may reflect an overall resistance of established pathology to treatment, age-associated differences in microglia activation [2], or a shifting phenotype of microglia in different stages of AD course [18]. Indeed, other investigators have shown that microglial inflammation pathways are robustly altered in $\operatorname{Tg} 4510$ mice and that these changes are detectible in young 4.7-month-old animals [41]. These age-associated and disease course-associated changes in microglia physiology indicate an important consideration for development of treatment strategies and suggest an important role for early intervention.

\section{Conclusions}

In summary, while microglia are attractive targets in Alzheimer's disease considering their contribution to the inflammatory response in disease, it is clear from these studies that a modest reduction in microglia is unlikely to change the outcome of late-stage tau pathology or reduce inflammatory gene expression. It is possible that a more severe depletion of microglia may lead to a change in neurodegeneration-related phenotypes, or that the effect of microglia is more marked on early phases of disease, prior even to the accumulation of tau aggregates in the brain. Thus, it is critical that we continue to investigate the specific factors responsible for these pathological alterations to understand the potential window of microglial intervention in both aging and disease. 


\section{Abbreviations}

A $\beta$ : Amyloid $\beta$; CSF1R: Colony stimulating factor 1 R; FRET: Fluorescence resonance energy transfer; HEK: Human embryonic kidney; IFD: Integrated FRET density; PBS: Phosphate-buffered saline; PLX: PLX3397; TBS: Tris-buffered saline; TNFa: Tumor necrosis factor a

\section{Acknowledgments}

We would like to acknowledge the donors of Alzheimer's Disease Research, a program of BrightFocus Foundation. We would also like to thank Abbvie Inc. for generously donating the PLX3397 for use in these experiments. Finally, we would like to acknowledge the support of the McLaughlin Research Institute including Rose Pitstick and Dr. George Carlson for their assistance with Tg4510 mice.

\section{Funding}

This work was supported by the Massachusetts Center for Alzheimer's Therapeutic Studies (MassCATS), a cooperative agreement with the Commonwealth of Massachusetts supporting precompetitive Alzheimer's disease research (SCH), National Institutes of Health NIA T32 AG000222, Martin L. and Sylvia Seevak Hoffman Fellow-Stewardship and by the BrightFocus Foundation (REB), National Institutes of Health P50 AG005134 (BTH), National Institutes of Health R01 AG059789-01 (BTH), and the JPB Foundation (BTH).

\section{Availability of data and materials}

The datasets used and/or analyzed during the current study are available from the corresponding author on reasonable request.

\section{Authors' contributions}

$\mathrm{SCH}$ and REB designed the study with the assistance of BTH. SCH, REB, ABR, $A B$, and $M H$ performed the experiments. SCH and REB analyzed and interpreted the data. REB and SCH wrote the manuscript with the assistance of BTH and ABR. All of the authors approved the final manuscript.

\section{Ethics approval}

All animal experiments were performed in accordance with national and institutional guidelines and were approved by the Massachusetts General Hospital and McLaughlin Research Institute Institutional Animal Care and Use Committees.

\section{Consent for publication}

N/A

\section{Competing interests}

The authors declare that they have no competing interests.

\section{Publisher's Note}

Springer Nature remains neutral with regard to jurisdictional claims in published maps and institutional affiliations.

\section{Author details}

${ }^{1}$ Department of Neurology, MassGeneral Institute for Neurodegenerative Disease, Massachusetts General Hospital, Harvard Medical School, Charlestown, MA 02129, USA. ${ }^{2}$ Biggs Institute for Alzheimer's and Neurodegenerative Disease, University of Texas Health Science Center San Antonio, 7703 Floyd Curl Drive, San Antonio, TX 78229, USA. ${ }^{3}$ Department of Pharmacology, University of Texas Health Science Center San Antonio, San Antonio, TX 78229, USA.

Received: 6 September 2018 Accepted: 26 October 2018

\section{Published online: 09 November 2018}

\section{References}

1. Asai H, Ikezu S, Tsunoda S, Medalla M, Luebke J, Haydar T, Wolozin B, Butovsky O, Kügler S, Ikezu T. Depletion of microglia and inhibition of exosome synthesis halt tau propagation. Nat Neurosci. 2015;18:1584-93.

2. Bardou I, Kaercher RM, Brothers HM, Hopp SC, Royer S, Wenk GL. Age and duration of inflammatory environment differentially affect the neuroimmune response and catecholaminergic neurons in the midbrain and brainstem. Neurobiol Aging. 2014;35:1065-73.
3. Bellucci A, Bugiani O, Ghetti B, Spillantini MG. Presence of reactive microglia and neuroinflammatory mediators in a case of frontotemporal dementia with P301S mutation. Neurodegener Dis. 2011;8:221-9.

4. Bennett RE, Robbins AB, Hu M, Cao X, Betensky RA, Clark T, Das S, Hyman BT. Tau induces blood vessel abnormalities and angiogenesis-related gene expression in P301L transgenic mice and human Alzheimer's disease. Proc Natl Acad Sci USA. 2018;115:E1289-98.

5. Bhaskar K, Konerth M, Kokiko-Cochran ON, Cardona A, Ransohoff RM, Lamb BT. Regulation of tau pathology by the microglial Fractalkine receptor. Neuron. 2010;68:19-31.

6. Byrne PV, Guilbert LJ, Stanley ER. Distribution of cells bearing receptors for a colony-stimulating factor (CSF-1) in murine tissues. J Cell Biol. 1981;91:848-53.

7. Carroll JA, Race B, Williams K, Striebel J, Chesebro B. Microglia are critical in host defense against prion disease Dermody TS, ed. J Virol. 2018;92.

8. Dagher NN, Najafi AR, Kayala KMN, Elmore MRP, White TE, Medeiros R, West $\mathrm{BL}$, Green KN. Colony-stimulating factor 1 receptor inhibition prevents microglial plaque association and improves cognition in 3xTg-AD mice. J Neuroinflammation. 2015;12:139.

9. Efthymiou AG, Goate AM. Late onset Alzheimer's disease genetics implicates microglial pathways in disease risk. Mol Neurodegener. 2017;12:43.

10. Elmore MRP, Najafi AR, Koike MA, Dagher NN, Spangenberg EE, Rice RA, Kitazawa M, Matusow B, Nguyen H, West BL, Green KN. Colony-stimulating factor 1 receptor signaling is necessary for microglia viability, unmasking a microglia progenitor cell in the adult brain. Neuron. 2014;82(2):380-97.

11. Ghosh S, Wu MD, Shaftel SS, Kyrkanides S, Laferla FM, Olschowka JA, O'Banion MK. Sustained interleukin-1 $\beta$ overexpression exacerbates tau pathology despite reduced amyloid burden in an Alzheimer's mouse model. J Neurosci. 2013;33:5053-64.

12. Hilla AM, Diekmann H, Fischer D. Microglia are irrelevant for neuronal degeneration and axon regeneration after acute injury. J Neurosci. 2017:37:6113-24.

13. Holmes BB, Furman JL, Mahan TE, Yamasaki TR, Mirbaha H, Eades WC, Belaygorod L, Cairns NJ, Holtzman DM, Diamond MI. Proteopathic tau seeding predicts tauopathy in vivo. Proc Natl Acad Sci U S A. 2014;111: E4376-85.

14. Hopp SC, Lin Y, Oakley D, Roe AD, DeVos SL, Hanlon D, Hyman BT. The role of microglia in processing and spreading of bioactive tau seeds in Alzheimer's disease. J Neuroinflammation. 2018;15:269.

15. Huang $Y, X u Z$, Xiong $S$, Sun F, Qin G, Hu G, Wang J, Zhao L, Liang $Y-X$, Wu T, Lu Z, Humayun MS, So K-F, Pan Y, Li N, Yuan T-F, Rao Y, Peng B. Repopulated microglia are solely derived from the proliferation of residual microglia after acute depletion. Nat Neurosci. 2018;21:530-40.

16. Jiang T, Zhang Y-D, Chen Q, Gao Q, Zhu X-C, Zhou J-S, Shi J-Q, Lu H, Tan L, Yu J-T. TREM2 modifies microglial phenotype and provides neuroprotection in P301S tau transgenic mice. Neuropharmacology. 2016;105:196-206.

17. Jin W-N, Shi SX-Y, Li Z, Li M, Wood K, Gonzales RJ, Liu Q. Depletion of microglia exacerbates postischemic inflammation and brain injury. J Cereb Blood Flow Metab. 2017:37:2224-36.

18. Keren-Shaul H, Spinrad A, Weiner A, Matcovitch-Natan O, Dvir-Szternfeld R, Ulland TK, David E, Baruch K, Lara-Astaiso D, Toth B, Itzkovitz S, Colonna M, Schwartz M, Amit I. A unique microglia type associated with restricting development of Alzheimer's disease. Cell. 2017;169:1276-90 e17.

19. Kitazawa M, Cheng D, Tsukamoto MR, Koike MA, Wes PD, Vasilevko V, Cribbs $\mathrm{DH}$, LaFerla FM. Blocking IL-1 signaling rescues cognition, attenuates tau pathology, and restores neuronal -catenin pathway function in an Alzheimer's disease model. J Immunol. 2011;187:6539-49.

20. Klein D, Patzkó Á, Schreiber D, van Hauwermeiren A, Baier M, Groh J, West BL, Martini R. Targeting the colony stimulating factor 1 receptor alleviates two forms of Charcot-Marie-Tooth disease in mice. Brain. 2015;138:3193-205.

21. Krasemann S, et al. The TREM2-APOE pathway drives the transcriptional phenotype of dysfunctional microglia in neurodegenerative diseases. Immunity. 2017:47:566-81.e9.

22. Lee M, McGeer E, McGeer PL. Activated human microglia stimulate neuroblastoma cells to upregulate production of beta amyloid protein and tau: implications for Alzheimer's disease pathogenesis. Neurobiol Aging. 2015;36:42-52.

23. Lee $\mathrm{S}$, Shi $X Q$, Fan $A$, West B, Zhang J. Targeting macrophage and microglia activation with colony stimulating factor 1 receptor inhibitor is an effective strategy to treat injury-triggered neuropathic pain. Mol Pain. 2018;14: 174480691876497. 
24. Li Y, Liu L, Barger SW, Griffin WST. Interleukin-1 mediates pathological effects of microglia on tau phosphorylation and on synaptophysin synthesis in cortical neurons through a p38-MAPK pathway. J Neurosci. 2003;23:1605-11.

25. Liddelow SA, Guttenplan KA, Clarke LE, Bennett FC, Bohlen CJ, Schirmer L, Bennett ML, Münch AE, Chung W-S, Peterson TC, Wilton DK, Frouin A, Napier BA, Stevens B, Barres BA. Neurotoxic reactive astrocytes are induced by activated microglia HHS public access. Nature. 2017;26:481-7.

26. Liu X, Yamashita T, Chen Q, Belevych N, Mckim DB, Tarr AJ, Coppola V, Nath N, Nemeth DP, Syed ZW, Sheridan JF, Godbout JP, Zuo J, Quan N. Interleukin 1 type 1 receptor restore: a genetic mouse model for studying interleukin 1 receptor-mediated effects in specific cell types. J Neurosci. 2015;35:2860-70

27. Luo W, Liu W, Hu X, Hanna M, Caravaca A, Paul SM. Microglia internalization and degradation of pathological tau is enhanced by an antitau monoclonal antibody. Sci Rep. 2015:5:11161.

28. Majerova P, Zilkova M, Kazmerova Z, Kovac A, Paholikova K, Kovacech B, Zilka N, Novak M. Microglia display modest phagocytic capacity for extracellular tau oligomers. J Neuroinflammation. 2014;11:161.

29. Maphis N, Jiang S, Xu G, Kokiko-Cochran ON, Roy SM, Van Eldik LJ, Watterson DM, Lamb BT, Bhaskar K. Selective suppression of the a isoform of p38 MAPK rescues late-stage tau pathology. Alzheimers Res Ther. 2016:8:54.

30. Maphis N, Xu G, Kokiko-Cochran ON, Jiang S, Cardona A, Ransohoff RM, Lamb BT, Bhaskar K. Reactive microglia drive tau pathology and contribute to the spreading of pathological tau in the brain. Brain. 2015;138:1738-55.

31. Patel S, Player MR. Colony-stimulating factor-1 receptor inhibitors for the treatment of cancer and inflammatory disease. Curr Top Med Chem. 2009;9:599-610.

32. Quintanilla RA, Orellana DI, González-Billault C, Maccioni RB. Interleukin-6 induces Alzheimer-type phosphorylation of tau protein by deregulating the cdk5/p35 pathway. Exp Cell Res. 2004;295:245-57.

33. SantaCruz K, Lewis J, Spires T, Paulson J, Kotilinek L, Ingelsson M, Guimaraes A, DeTure M, Ramsden M, McGowan E, Forster C, Yue M, Orne J, Janus C, Mariash A, Kuskowski M, Hyman B, Hutton M, Ashe KH. Tau suppression in a neurodegenerative mouse model improves memory function. Science. 2005;309:476-81.

34. Sasaki A, Kawarabayashi T, Murakami T, Matsubara E, Ikeda M, Hagiwara H, Westaway D, George-Hyslop PS, Shoji M, Nakazato Y. Microglial activation in brain lesions with tau deposits: comparison of human tauopathies and tau transgenic mice TgTauP301L. Brain Res. 2008;1214: 159-68.

35. Serrano-Pozo A, Gómez-Isla T, Growdon JH, Frosch MP, Hyman BT. A phenotypic change but not proliferation underlies glial responses in Alzheimer disease. Am J Pathol. 2013;182:2332-44.

36. Serrano-Pozo A, Mielke ML, Gómez-Isla T, Betensky RA, Growdon JH, Frosch MP, Hyman BT. Reactive glia not only associates with plaques but also parallels tangles in Alzheimer's disease. Am J Pathol. 2011;179:1373-84.

37. Sosna J, Philipp S, Albay R, Reyes-Ruiz JM, Baglietto-Vargas D, LaFerla FM, Glabe CG, Glabe CG. Early long-term administration of the CSF1R inhibitor PLX3397 ablates microglia and reduces accumulation of intraneuronal amyloid, neuritic plaque deposition and pre-fibrillar oligomers in 5XFAD mouse model of Alzheimer's disease. Mol Neurodegener. 2018;13:11.

38. Spangenberg EE, Lee RJ, Najafi AR, Rice RA, Elmore MRP, Blurton-Jones M, West BL, Green KN. Eliminating microglia in Alzheimer's mice prevents neuronal loss without modulating amyloid- $\beta$ pathology. Brain. 2016;139: 1265-81.

39. Szalay G, Martinecz B, Lénárt N, Környei Z, Orsolits B, Judák L, Császár E, Fekete R, West BL, Katona G, Rózsa B, Dénes Á. Microglia protect against brain injury and their selective elimination dysregulates neuronal network activity after stroke. Nat Commun. 2016;7:11499.

40. Tosto G, Reitz C. Genome-wide association studies in Alzheimer's disease: a review. Curr Neurol Neurosci Rep. 2013:13:381.

41. Wes PD, et al. Tau overexpression impacts a neuroinflammation gene expression network perturbed in Alzheimer's disease. PLoS One. 2014;9: e106050.

42. Yang X, Ren H, Wood K, Li M, Qiu S, Shi F-D, Ma C, Liu Q. Depletion of microglia augments the dopaminergic neurotoxicity of MPTP. FASEB J. 2018; 32:3336-45.

\section{Ready to submit your research? Choose BMC and benefit from:}

- fast, convenient online submission

- thorough peer review by experienced researchers in your field

- rapid publication on acceptance

- support for research data, including large and complex data types

- gold Open Access which fosters wider collaboration and increased citations

- maximum visibility for your research: over $100 \mathrm{M}$ website views per year

At BMC, research is always in progress.

Learn more biomedcentral.com/submissions 